\title{
Commissioning Status of NSLS-II Vacuum Control
}

\section{System}

\author{
Huijuan Xu, Hsiao-chaun Hseuh, Shuwei Leng and Douglas T Zigrosser \\ Photon Science Directorate, BNL, Upton, NY 11973, U.S.A.
}

\begin{abstract}
The NSLS-II (National Synchrotron Light Source II) is a state-of-the-art $3 \mathrm{GeV}$ third generation light source currently under integrated testing and commissioning at Brookhaven National Laboratory. The vacuum systems are monitored by vacuum gauges and ion pump current. The gate valves are controlled by PLC (programmable logic controllers) using voting scheme. EPICS (Experimental Physics and Industrial Control System) application codes provide the high level monitoring and control through the input-output controllers. This paper will discuss the commissioning status of the various aspects of vacuum control system.
\end{abstract}

Key words: NSLS-II, vacuum, control system, commissioning status.

\section{Introduction}

The NSLS-II (National Synchrotron Light Source II) accelerator complex comprises a $200 \mathrm{MeV}$ linac, LTB (linac-to-booster transport line), a $3 \mathrm{GeV}$ BR (booster ring), BTS (booster-to-storage ring transport line), and the $3 \mathrm{GeV}$ SR (storage ring). The booster ring and the storage ring share the same tunnel. The booster ring is divided into four arc sections and four straight sections. The storage ring vacuum systems are divided into 60 vacuum sectors with RF-shielded GV (gate valves) mounted at both ends of each straight section. The vacuum system includes all vacuum chambers, vacuum pumps, vacuum instrumentation and diagnostics, vacuum controllers, and connecting wiring. The vacuum control system will interface with vacuum devices while being part of the machine control [1]. There are also front end and beam line vacuum systems which are not included in this paper.

\section{NSLS-II Vacuum Components}

Major components used in NSLS-II vacuum system are listed in Table 1. Nominal pumping speed of over

Corresponding author: Huijuan $\mathrm{Xu}$, Ph.D., research fields: accelerator vacuum \& diagnostic control. E-mail: hxu@bnl.gov.
$100,000 \mathrm{l} / \mathrm{s}$ is provided by ion pumps, titanium pumps and NEG strips for the ring [2].

These components are controlled by corresponding controllers installed in mezzanine racks, which are listed in Table 2.

\section{NSLS-II Vacuum Control System}

NSLS-II vacuum control system interfaces with VGC (gauges controllers), IPC (ion pump controllers), TSPC (Titanium pump controllers), chamber and absorber temperature readouts, cooling water flow sensors, gate valve limit switches, fast valve (FV) sensors and controllers (FVC) to monitor and control the vacuum components listed in Table 1.

NSLS-II vacuum control system is a subsystem of NSLS-II control system and follows the architecture adopted by NSLS-II control system. The core of the EPICS (Experimental Physics and Industrial Control System) [3] has been chosen as the basis for the NSLS-II control system.

Based on EPICS, the vacuum control system has three layers: OPI (operator interfaces), IOC (input output controllers) and device controllers. Fig. 1 shows the architecture of NSLS-II vacuum control system. 
Table 1 Major vacuum components in NSLS-II.

\begin{tabular}{llllllll}
\hline & IP & CCG & TCG & GV & TSP & RGA & FV \\
\hline Linac & 23 & 6 & 6 & 6 & - & - & - \\
LTB & 13 & 6 & 4 & 4 & - & - & - \\
BR & 78 & 17 & 9 & 9 & - & 4 & - \\
BTS & 15 & 8 & 5 & 5 & - & - & 1 \\
SR & 225 & 120 & 60 & 60 & 225 & 38 & - \\
\hline
\end{tabular}

Table 2 Vacuum controllers in NSLS-II.

\begin{tabular}{lllllll}
\hline & IPC & VGC & PLC & TSPC & RGAC & FVC \\
\hline Linac & 12 & 3 & 1 & - & - & - \\
LTB & 7 & 3 & 1 & - & - & - \\
BR & 40 & 9 & 4 & - & 2 & - \\
BTS & 8 & 4 & 1 & - & - & 1 \\
SR & 120 & 60 & 30 & 225 & 38 & - \\
\hline
\end{tabular}

\subsection{Operator Interface}

The operator interface provides access and control to NSLS-II vacuum control system. The overall screen gives entry to each subsystem of NSLS-II accelerator complex. The details of each controlled item are provided in the subsystem screens. A vacuum monitoring screen has been developed to provide a full and accurate understanding of NSLS-II vacuum system.

The EPICS toolkit provides a suite of applications for the OPI. NSLS-II uses CSS (Control System Studio) [4] as the main operator application.

The CSS is an Eclipse-based collection of tools to monitor and operate large scale control systems, such as the ones widely used in the accelerator community. It's an excellent platform for EPICS control system and provides many tools, such as BOY (Best OPI Yet), BEAST (Best Ever Alarm System Toolkit), data browser, etc. BOY is an operator interface development and runtime environment. BEAST will be used as a replacement of ALH (Alarm Handler) [5]. Data browser is a trending tool and provides easy way to view, retrieve archived data. All data communication between OPIs and IOCs is accomplished through the EPICS CA (Channel Access) protocol [6] over Ethernet.

Channel Archiver [7] is used as the data logging system to $\log$ all important parameters, such as pressure readings, RTD temperature readings, GV status, and so on.

IRMIS (Integrated Relational Model of Installed Systems) [8] will be used to describe all components and wiring data. Data can be further served up to web pages through a CGI (Common Gateway Interface) server.

\subsection{Input/Output Controller}

Vacuum IOC supports the process control for all vacuum devices and provides all information for machine operator. Cost-effective IBM server computers are used for that purpose.

The server provides distributed processing and concentrates the control activities of sets or assemblies of equipment or entire sub-processes. 6 IBM servers are dedicated for vacuum system, one for injector system, and one for each storage ring pentant. All 6 servers are placed in ring building control room. These IOCs communicate with vacuum devices through local network, and with other systems via the control network.

\subsection{Vacuum Devices Control}

The vacuum control system interfaces directly with each vacuum device as seen in Fig. 1, and as part of the storage ring machine protection systems (EPS). Due to the high radiation levels, all vacuum controllers are located in injection service area and in SR mezzanine. The VGC, PLC and communications equipment are backed with uninterruptible power supplies. These vacuum devices can be operated via front-panel display and with the machine control system through RS-232 or Ethernet for high-level monitoring and control. The terminal servers, MOXA CN2650I-16-2AC are used to integrate various vacuum devices. This module comes with 16 serial ports and provides $2 \mathrm{KV}$ optical isolation on each port. 37 terminal servers will be used for initial operation, 1 for each injector area and each storage ring cell. 


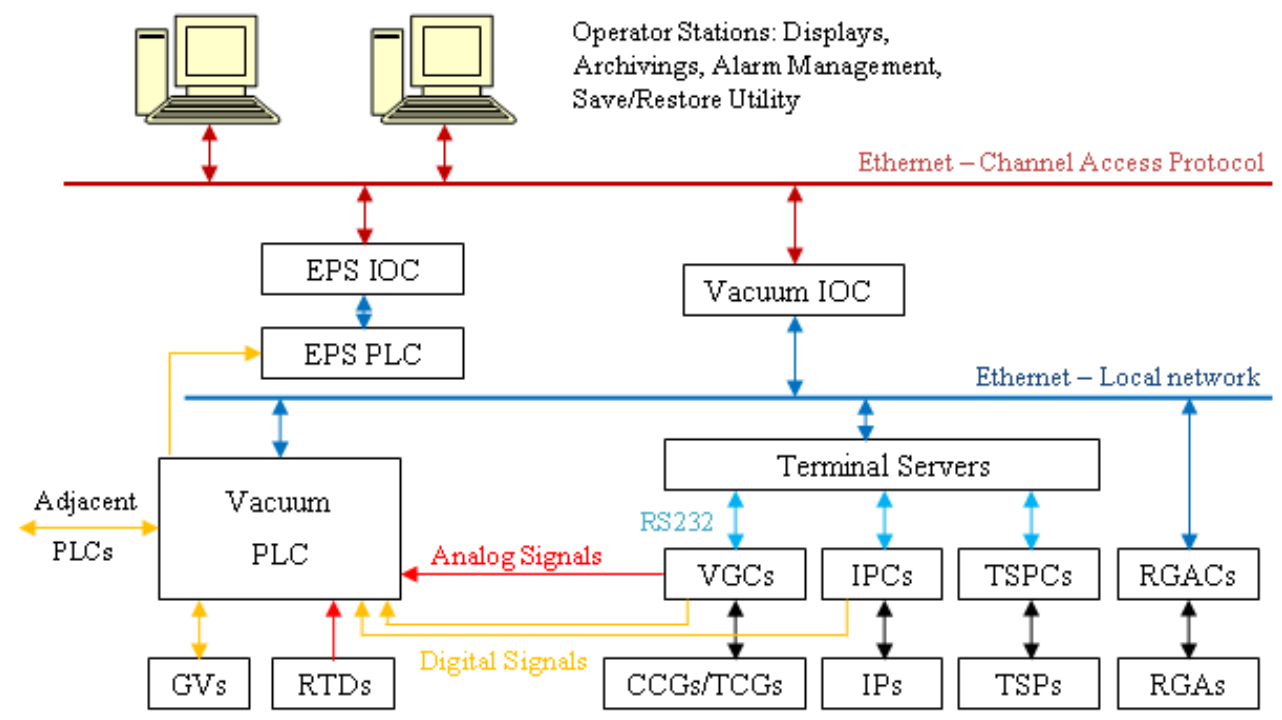

Fig. 1 NSLS-II vacuum control system architecture.

The PLCs use a voting scheme, with inputs from the set point contacts of several gauges and ion pumps, to initiate valve interlock and closure. The voting scheme was implemented to minimize false triggering due to the malfunctioning of a single gauge or pump. The fast response analogue CCG signal from the gauge controller is used for machine protection system. Each PLC is equipped with a Panel View for local diagnostic and troubleshooting.

\section{Current Commissioning Status}

\subsection{Linac}

The Linac vacuum control system was originally developed by RI (Research Instruments) and was designed to meet NSLS-II vacuum specifications. A Simens PLC is used to interface with all vacuum devices and provide interlock signals to the vacuum system and EPS. The PLC system monitors IPs HV status, CCG relay status, CCG analogue readings, and controls the GVs. A terminal server was added later to interface with all vacuum controllers through serial ports.

The operator interface to the Linac vacuum system is implemented with EDM and BOY. The PLC system was developed by RI and is displayed in EDM (Extensible Display Manager) [9] screens and provides useful information for vacuum diagnostic. The BOY screens were created to provide more detailed information of vacuum devices. Fig. 2 shows the main screen for the Linac vacuum control system. Real-time readings of all CCGs, IPs, GVs as well as the average pressure values of each section between GVs are monitored and displayed in the main Linac vacuum screen. Device icons in main Linac vacuum screen give entry to each vacuum device. The device screens provide monitor and control to corresponding vacuum devices.

The live readings of CCGs and IPs are grouped together and displayed in bar format in a separate screen giving a better visual display. Historical plot is also provided in different screens to view the logged data, e.g. pressure readings and GV status values.

\subsection{LTB, Booster and BTS}

The LTB, Booster, and BTS vacuum control system was developed together by BINP (Budker Institute of Nuclear Physics) and BNL. The terminal servers are used to integrate various vacuum devices through serial ports. The Allen Bradley PLCs are used to interlock the vacuum system and EPS. The PLC system provides monitor on IPs relay status, CCGs relay status, CCG analogue readings, and control to GVs. 


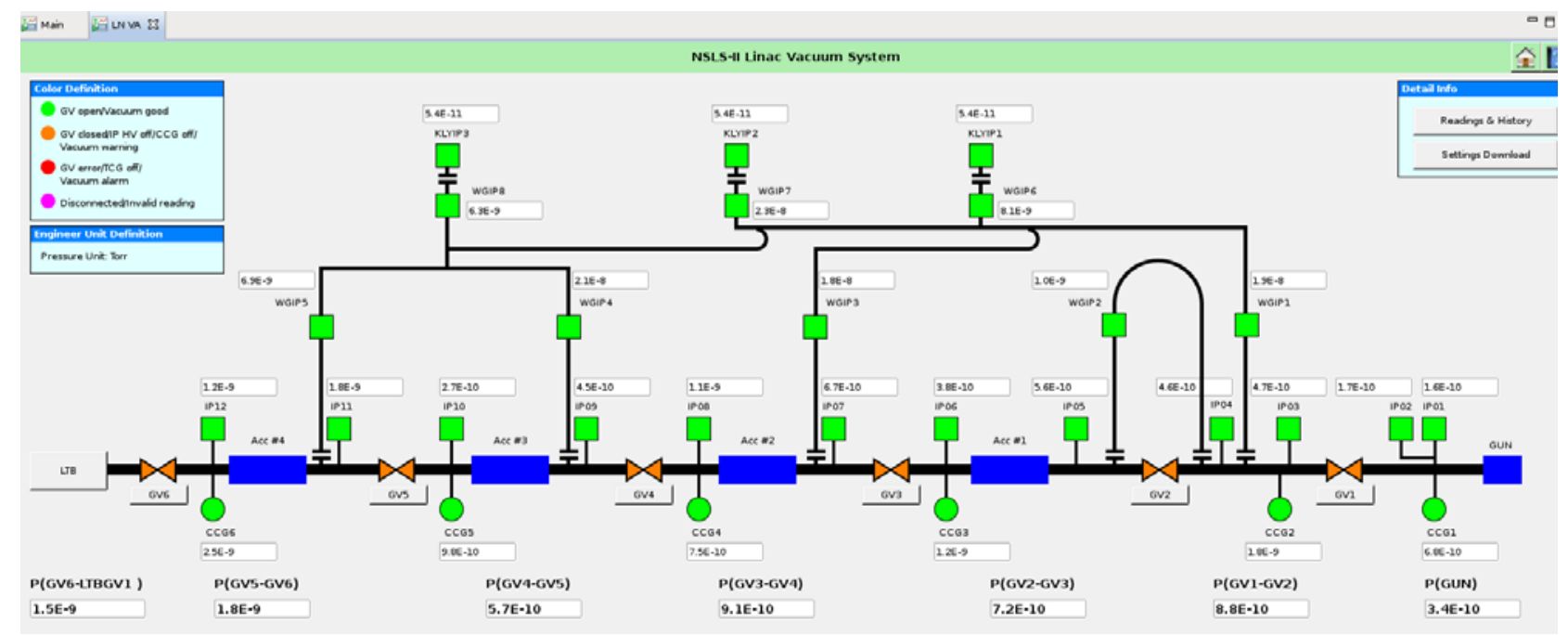

Fig. 2 NSLS-II Linac vacuum control system main panel.

The PLCs use a voting scheme, with setpoint relay inputs from 2 IPs and 1 CCG. If the pressure readings of two out of three devices in the logic exceed the set point value of $2 \times 10^{-6}$ Torr, the GVs at both sides of the concerning section are closed immediately. TCGs are used to interlock IPs from turning on through EPICS.

One IBM computer running Debian is installed in injector vacuum rack for local diagnostic and troubleshooting.

Pressure readings from CCGs, IPs are logged at 1 Hz. Average pressures for each section are logged at the same rate.

\subsection{Storage Ring}

The configuration for vacuum control related devices have been completed for all cells in storage ring. These devices including CCGs, TCGs, IPs and RGAs have been turned on for monitored and controlled through EPICS, following the commissioning of the vacuum sectors.

The PLC logic has been tested through EPICS and CSS panels. The PLCs use a similar voting scheme as Booster Ring, with set point relay inputs from 2 CCGs and 1 IP. If pressure readings of two out of three devices in the logic exceed the set point value $2 \times 10^{-7}$ Torr, the GVs at both sides of the concerning section are closed immediately, as well as the gate valves at adjacent cells. The relay signals from TCG are used for GV interlock as well as IP protection.

Ten IBM servers, one out of every three cells, have been installed at vacuum rack to provide local diagnostic and troubleshooting.

Pressure readings from CCGs, IPs are logged at $1 \mathrm{~Hz}$, as well as average pressures for each section and each cell. RGAs are constantly run PROFILE scan of 1-100 amu with a 0.13 step size under EPICS. Important parameters affecting the operation of RGA are constantly monitored and archived, such as filament status, emission status, over pressure trip, and measurement values [10]. The TSPs are used during maintenance period.

\subsection{Front End}

Six front ends have been built along with the initial six project beam lines. These front ends adopt the standard vacuum devices, e.g. CCGs, TCGs, IPs, TSPs and RGAs. The interlock is implemented through PLC with the same voting scheme from 2 CCGs and 1 IP. One FV and two FV sensors are installed in each front end. The FV is provided to intercept the pressure shock wave in the case of a vacuum breach in the beamline which is to shut within a few milliseconds once triggered by FV sensors. 
The important vacuum parameters are archived at the same rate as other subparts. Till now, all these front ends have been fully tested and put into normal operation for more than one year.

\section{Conclusions}

The NSLS-II accelerator was successfully commissioned during the spring and summer of 2014. It achieved the first electron beam in April 2014 and reached $25 \mathrm{~mA}$ of current at 3-Gev in the storage ring with new RF cavity in July 2014. On July 11, 2014, the NSLS-II team achieved a store beam of $50 \mathrm{~mA}$. The EPICS based vacuum control system performs well and provides useful information for accelerator physicists and operators during the commissioning process. The control group and vacuum group continue to work closely to develop a reliable, user friendly control system.

\section{References}

[1] NSLS-II Preliminary Design Report, 2008, Brookhaven National Laboratory, 2008. Accessed June 12, 2015,
http://www.bnl.gov/nsls2/project/PDR.

[2] Hseuh, H. C., Blednykh, A., Doom, L., Ferreira, M., Hetzel, C., Hu, J. P., and et al. 2009. "Status of NSLS-II Vacuum System.” PAC 2009, 348.

[3] EPICS, Argonne National Laboratory, Accessed June 12, 2015, http://www.aps.anl.gov/epics.

[4] CSS, Brookhaven National Laboratory, Accessed June 12, 2015, http://cs-studio.sourceforge.net.

[5] ALH, Argonne National Laboratory, Accessed June 12, 2015,http://www.aps.anl.gov/epics/extensions/alh/index.p hp

[6] Channel Access, Argonne National Laboratory, Accessed June 12, 2015,

http://www.aps.anl.gov/epics/base/R3-14/8-docs/CAref.h tml

[7] Channel Archiver, Kay Kasemir, Nikolay Malitsky. Accessed June 12, 2015, http://sourceforge.net/apps/trac/epicschanarch/wiki

[8] IRMIS, Brookhaven National Laboratory, Accessed June 12, 2015, http://cs-studio.sourceforge.net.

[9] EDM, Oak Ridge National Laboratory, Accessed June 12, 2015, http://ics-web.sns.ornl.gov/edm/

[10] Xu, H., Hseuh, H., Wilson, K., Zigrosser, D. T., and Ferreira, M. J. 2013. "EPICS Interface and Control of NSLS-II Residual Gas Analyzer System.” In Proceedings of ICALEPCS2013, MOPPC122, San Francisco, CA, USA. 